\title{
INERT EXTENSIONS OF KRULL DOMAINS
}

\author{
DOUGLAS L. COSTA AND JON L. JOHNSON
}

\begin{abstract}
Let $A \subseteq B$ be integral domains with $B$ an inert extension of a Krull domain $A$. Let $\mathscr{P}(A)$ be the set of height one primes of $A$, and let $T=\cap_{p \in \mathscr{T}(A)} B \otimes A_{p}$. When each $B_{p}=B \otimes A_{p}$ is a UFD, a necessary and sufficient condition for $T$ to be a Krull domain is obtained. If $T$ is a Krull domain and each $B_{p}$ is a UFD, then the divisor class groups of $A$ and $T$ are isomorphic under the natural mapping.

These results are applied to $A \subseteq B$ when $B$ is a symmetric algebra over $A$ and when $B$ is locally a polynomial ring over $A$.
\end{abstract}

Let $A \subseteq B$ be integral domains. Following Cohn [3] we say that $B$ is an inert extension of $A$ if for any nonzero $x, y \in B, x y \in A$ implies $x \in A, y \in A$. If $B$ is an inert extension of $A$ and a Krull domain, then $A$ is easily seen to be a Krull domain. In this paper we give a partial converse to this result, and we compare the class groups of $A$ and $B$, when both are Krull domains.

This paper originated as a study of extensions $A \subseteq B$ with $A$ a Krull domain and $B$ a symmetric algebra over $A$. Under these hypotheses it is known that when $A$ is a UFD, $B$ is a UFD if and only if $B$ is a factorial $A$ module [4], [5]. An $A$-module $M$ over a UFD $A$ is factorial if it is torsion-free and every element $x \in M$ may be written uniquely $x=a x^{*}$, with $a$ $\in A$ and $x^{*}$ an element of $M$ divisible only by units of $A$. Elements of $M$ divisible only by units are called primitive.

We generalize the idea of a factorial module as follows. Let $A$ be a Krull domain, $M$ an $A$-module, and $\mathcal{P}(A)$ the set of height one primes of $A$. We say that $M$ is a Krull $A$-module if $M$ is torsion-free, $M_{p}=M \otimes_{A} A_{p}$ is a factorial $A_{p}$-module for each $p \in \mathscr{P}(A), M=\cap_{p \in \mathscr{P}(A)} M_{p}$, and each nonzero $x$ in $M$ is primitive in all but a finite number of $M_{p}$ (i.e. the intersection is "locally finite"). Our main result, Theorem 1.6, shows that if $A \subseteq B$ is an inert extension with $A$ a Krull domain and $B_{p}$ a UFD for each $p \in \mathscr{P}(A)$, then $T=\bigcap_{p \in \mathscr{P}(A)} B_{p}$ is a Krull domain if and only if it is a Krull $A$-module, in which case $\mathrm{Cl}(A) \cong \mathrm{Cl}(T)$. In the second section we show that in the case when $B$ is a symmetric algebra over $A$, the condition that $B_{p}$ be a UFD for each $p \in \mathscr{P}(A)$ is superfluous. We conclude with some examples.

We owe a debt of originality to $\$ 10$ of [7], as our main results are generalizations of Theorem 10.11 of [7] and our arguments, for the most part,

Received by the editors February 13, 1976.

AMS (MOS) subject classifications (1970). Primary 13F05; Secondary 13B99.

Key words and phrases. Krull domain, divisor class group, polynomial ring, symmetric algebra. C American Mathematical Society 1976 
are adaptations of those of [7]. Indeed, one might consider this paper a supplement to [7].

1. For a Krull domain $A$ let $\operatorname{Div}(A)$ be the group of divisorial fractional ideals of $A$, Prin $(A)$ the group of principal fractional ideals of $A, \mathrm{Cl}(A)$ $=\operatorname{Div}(A) /$ Prin $(A)$ the divisor class group of $A$, and $\mathscr{P}(A)$ the set of height one prime ideals of $A$. If $A \subseteq B$ are Krull domains we say that (PDE) holds if $P \in \mathscr{P}(B)$ implies $P \cap A \in \mathscr{P}(A) \cup\{0\}$. When (PDE) holds, the map $\operatorname{Div}(A) \rightarrow \operatorname{Div}(B)$ given by $\mathfrak{A} \rightarrow B:(B: \mathfrak{A} B)$ is a homomorphism [7, Proposition 6.3(d)] which clearly maps Prin $(A)$ into Prin $(B)$, and so induces a natural homomorphism $\mathrm{Cl}(A) \rightarrow \mathrm{Cl}(B)$. We begin by investigating this map when $A \subseteq B$ is an inert extension.

Lemma 1.1. Let $A \subseteq B$ be an inert extension of integral domains and let $\mathfrak{A}$ be an ideal of $A$. Then $A:(A: \mathfrak{U}) \supseteq(B:(B: \mathfrak{U} B)) \cap A$.

Proof. Let $x \in A$ and suppose $x(B: \mathfrak{A} B) \subseteq B$. If $y \in A: \mathfrak{A}$, then $y \in B$ : $\mathfrak{U} B$ and hence $x y \in B$. Say $x y=b$ and $y=a / t$ with $a, t \in A$. Then $t b=a x$ $\in A$, so $b \in A$ and hence $x \in A:(A: \mathfrak{U})$.

Proposition 1.2. Let $A \subseteq B$ be an inert extension of Krull domains for which (PDE) holds. Then $\mathrm{Cl}(A) \rightarrow \mathrm{Cl}(B)$ is injective.

Proof. We first show that $\operatorname{Div}(A) \rightarrow \operatorname{Div}(B)$ is injective. Since $\operatorname{Div}(A)$ and $\operatorname{Div}(B)$ are free abelian groups on the sets $\mathscr{P}(A)$ and $\mathscr{P}(B)$, it suffices to show that for each $p \in \mathscr{P}(A)$ there is a $P \in \mathscr{P}(B)$ such that $P \cap A=p$. But if $p$ is a prime divisorial ideal of $A$, then $p=(B:(B: p B)$ $\cap A)$ by the lemma. Hence $B:(B: p B)$ is a proper divisorial ideal of $B$ and is contained in some $P \in \mathscr{P}(B)$. Then $P \cap A=p$.

It remains to show that $\operatorname{Prin}(B) \cap \operatorname{Div}(A)=\operatorname{Prin}(A)$. But if $\mathscr{A}$ is a divisorial ideal of $A$ such that $B:(B: \mathfrak{A} B)$ is principal, then from $\mathfrak{A}$ $=(B:(B: \mathfrak{A} B)) \cap A$ and the inertness of the extension we deduce that $\mathfrak{A}$ is principal.

Lemma 1.3. Let $A \subseteq B$ be an extension of Krull domains for which (PDE) holds. If $\mathfrak{B}$ is a divisorial ideal in $B$ then $\mathfrak{B}=\bigcap_{p \in \varphi(A)} \mathfrak{B}_{p}$.

Proof. Suppose that $\mathfrak{B} \neq \bigcap_{p \in \mathscr{P}(A)} \mathfrak{B}_{p}$. For each element $x \in \cap \mathfrak{B}_{p} \backslash \mathfrak{B}$, $\left(\mathfrak{B}:{ }_{B} x\right)=\{y \in B \mid y x \in \mathfrak{B}\}$ is a divisorial ideal of $B$. Choose such an $x$ so that $\left(\mathfrak{B}:{ }_{B} x\right)$ is maximal in this family of divisorial ideals. To see that $\left(\mathfrak{B}:{ }_{B} X\right)$ is prime let $f, g \in B$ with $f g \in\left(\mathscr{B}:{ }_{B} x\right)$. If $g \notin\left(\mathfrak{B}:{ }_{B} x\right)$, then $g x$ $\in \cap \mathfrak{B}_{p} \backslash \mathfrak{B}$ and $\left(\mathfrak{B}:{ }_{B} g x\right) \supseteq\left(\mathfrak{B}:{ }_{B} x\right)+f B$. By the maximality of $\left(\mathfrak{B}:{ }_{B} x\right)$ we have $f \in\left(\mathscr{B}:{ }_{B} x\right)$. Let $P=\left(\mathscr{B}:{ }_{B} x\right)$. Being divisorial, $P$ is a height one prime of $B$ and hence there is a height one prime $p$ of $A$ such that $P \cap A \subseteq p$. Then $P_{p} \neq B_{p}$, but $P_{p}=\left(\mathfrak{B}:{ }_{B} x\right)_{p}=\left(\mathfrak{B}_{p}:{ }_{B_{p}} x\right)=B_{p}$, a contradiction. This proves the lemma.

THEOREM 1.4. Let $A \subseteq B$ be an inert extension of Krull domains for which (PDE) holds. Further suppose that $B_{p}$ is a UFD for each $p \in \mathscr{P}(A)$. Then the 
natural homomorphism $\mathrm{Cl}(A) \rightarrow \mathrm{Cl}(B)$ is an isomorphism.

Proof. In virtue of Proposition 1.2, we only need to prove that $\mathrm{Cl}(A)$ $\rightarrow \mathrm{Cl}(B)$ is surjective. By Nagata's Theorem [7, Theorem 7.1] it is sufficient to show that if $p \in \mathscr{P}(A)$, then the divisorial ideal $B:(B: p B)$ is prime in $B$. Let $q \in \mathscr{P}(A)$. Then $(B:(B: p B))_{q}=B_{q}:\left(B_{q}: p B_{q}\right)$; but $p B_{q}$ is principal so that $B_{q}:\left(B_{q}: p B_{q}\right)=p B_{q}$. By the lemma,

$$
B:(B: p B)=\bigcap_{q \in \mathcal{P}(A)}(B:(B: p B))_{q}=\bigcap_{q \neq p} B_{q} \cap p B_{p}=B \cap p B_{p}
$$

which is prime since $B_{p}$ is a UFD.

In the next section we give an example to show that the hypothesis that $B_{p}$ be a UFD for $p \in \mathscr{P}(A)$ cannot be deleted from Theorem 1.4.

To prepare for our next result we first need some equivalent forms of (PDE).

Proposition 1.5. Let $A \subseteq B$ be Krull domains. The following are equivalent:

(1) $A \subseteq B$ satisfies (PDE).

(2) $B=\bigcap_{p \in \mathcal{P}(A)} B_{p}$.

(3) Every $A$-sequence $a, b$ is a $B$-sequence.

Proof. We first show that (2) and (3) are equivalent with $B$ replaced by any torsion-free $A$-module $M$.

(2) $\Rightarrow(3)$. Let $a, b$ be an $A$-sequence and let $x, y \in M$ be such that $a x=b y$. Given $p \in \mathscr{P}(A)$, we have $(a, b) \nsubseteq p$ since $p$ is divisorial. If $b \notin p$ then $y \in a M_{p}$; and if $a \notin p$ then $y \in M_{p}=a M_{p}$. Hence

$$
y \in \bigcap_{p \in \mathcal{P}(A)} a M_{p}=a \bigcap_{p \in \mathcal{P}(A)} M_{p}=a M .
$$

Therefore $a, b$ is an $M$-sequence.

(3) $\Rightarrow(2)$. Let $x \in \cap M_{p}$. Then for each $p \in \mathcal{P}(A),(M: x)_{p}=\left(M_{p}: x\right)$ $=D_{p}$, so $(M: x) \nsubseteq p$. Let $a \in(M: x), a \neq 0$, and let $p_{1}, \ldots, p_{n}$ be the height one primes containing $a$. Then $a A=\cap_{i=1}^{n} Q_{i}$ with $Q_{i} p_{i}$-primary for $i=1$, $\ldots, n[8$, Corollary 43.10]. Denoting by $Z(A / a A)$ the set of zero-divisors on the module $A / a A$, we now have $Z(A / a A) \subseteq \cup_{i=1}^{n} p_{i}$ [1, Proposition 4.7]. Since $(M: x) \nsubseteq p_{i}$ for any $i$, we may choose $b \in(M: x) \backslash Z(A / a A)$. Then $a, b$ is an $A$-sequence and therefore an $M$-sequence. Since $b(a x)=a(b x)$ with $a x, b x$ $\in M$ we have $a x \in a M$ and hence $x \in M$. Thus $M=\cap M_{p}$.

(3) $\Rightarrow(1)$. Let $P \in \mathscr{P}(B)$ and set $p=P \cap A$. If ht $p>1$, pick $a \in p, a$ $=0$ and then choose $b \in p \backslash Z(A / a A)$ as in the proof of (3) $\Rightarrow$ (2). Then $a, b$ is an $A$-sequence and therefore a $B$-sequence in $P$, so ht $P \geqslant 2[9$, Theorem 131]. This contradicts ht $P=1$, so ht $p \leqslant 1$.

(1) $\Rightarrow$ (3). Let $a, b$ be an $A$-sequence. Since $B$ is a Krull domain, $a B=\bigcap_{i=1}^{n} Q_{i}$ with each $Q_{i} P_{i}$-primary for some $P_{i} \in \mathcal{P}(B)$. Hence $Z(B / a B)$ $\subseteq \cup_{i=1}^{n} P_{i}$. If $b \in Z(B / a B)$ we get $a, b \in P_{i}$ for some $i$. But then $a, b$ $\in P_{i} \cap A$ which implies that ht $\left(P_{i} \cap A\right) \geqslant 2$, contradicting (PDE). It follows that $b \notin Z(B / a B)$, i.e. that $a, b$ is a $B$-sequence.

Now for the main theorem. 
THEOREM 1.6. Let $A \subseteq B$ be an inert extension with $A$ a Krull domain and $B_{p}$ a UFD for each $p \in \mathscr{P}(A)$. Let $T=\cap B_{p}$. Then $A \subseteq T$ is an inert extension and $T$ is a Krull domain if and only if it is a Krull A-module, in which case $\mathrm{Cl}(T) \cong \mathrm{Cl}(A)$, by the natural homomorphism.

Proof. It is easy to see that $A \subseteq T$ is an inert extension. And if $T$ is a Krull domain then since $T=\cap T_{p}$, (PDE) holds for the extension $A \subseteq T$ by Proposition 1.5. The desired isomorphism of class groups now follows from Theorem 1.4. Thus it only remains to demonstrate the equivalence in the present theorem.

Suppose $T$ is a Krull domain. For $p \in \mathcal{P}(A), T_{p}=B_{p}$ is a UFD and an inert extension of the DVR $A_{p}$. Hence $T_{p}$ is a factorial $A_{p}$-module. It also follows that $p T_{p}$ is a height one prime of $T_{p}$, and so $p T_{p} \cap T$ is height one. It follows that if $f \in T, f \neq 0$, then $f$ belongs to only a finite number of the ideals $p T_{p} \cap T$. Thus $f$ is primitive as an element of the $A_{p}$-module $T_{p}$ for all but a finite number of $p$ in $\mathscr{P}(A)$.

Conversely, suppose that $T$ is a Krull $A$-module. Since $p T_{p}$ is a principal prime of the UFD $T_{p}=B_{p}, T_{p}=\left(T_{p}\right)_{p T_{p}} \cap T_{(A \backslash 0)}$. Thus

$$
T=\bigcap_{p \in \mathcal{P}(A)}\left(T_{p}\right)_{p T_{p}} \cap T_{(A \backslash 0)} .
$$

For each element $x$ in $T, x$ is primitive in all but a finite number of the $T_{p}$ implies that $x$ is a unit in all but a finite number of the $\left(T_{p}\right)_{p T_{p}}$. Hence by [14, Proposition 4.1], $\cap\left(T_{p}\right)_{p T_{p}}$ is a Krull domain and, since $T_{(A \backslash 0)}$ is a UFD, $T$ is a Krull domain.

Remarks. (1) In the next section we will give an example to show that, under the hypotheses of Theorem 1.6, $B$ may fail to be a Krull domain even though $T$ is one.

(2) If in Theorem 1.6, $B$ is a Krull domain, then $T$ is a subintersection of $B$ and hence is a Krull domain. In fact,

$$
T=\bigcap_{p \in \mathscr{P}(A)} B_{p}=\bigcap_{p \in \mathscr{P}(A)} \underset{P \in \mathscr{P}(B) ; P \cap A \subseteq p}{\bigcap_{P}} B_{P}=\bigcap_{\mathrm{ht}(P \cap A) \leqslant 1 ; P \in \mathscr{P}(B)} B_{P} .
$$

By Nagata's theorem [7, Theorem 7.1] there is an exact sequence of abelian groups $0 \rightarrow K \rightarrow \mathrm{Cl}(B) \rightarrow \mathrm{Cl}(T) \rightarrow 0$, where $K$ is generated by the divisor classes of height one primes $P$ of $B$ such that ht $(P \cap A)>1$. Note that this relates $\mathrm{Cl}(A)$ and $\mathrm{Cl}(B)$, since $\mathrm{Cl}(T) \cong \mathrm{Cl}(A)$. Furthermore, $K=0$ if and only if every $P \in \mathscr{P}(B)$ such that ht $(P \cap A)>1$ is principal. But if $P$ is principal in $B$ and $P \cap A \neq 0$ any generator for $P$ must lie in $A$, whence $P \cap A$ is principal and has height one. It follows that $K=0$ if and only if (PDE) holds for $A \subseteq B$, i.e. if and only if $B=T$.

(3) Eakin and Silver [6] examine extensions $A \subseteq B$ of integral domains in which $B$ is locally a polynomial ring over $A$. Since inertness is a local property, all such extensions are inert. If, moreover, $A$ is a Krull domain, $B=T[6$, (2.26)] and $B_{p}$ being a polynomial ring over $A_{p}$ is a UFD. From these 
observations one easily obtains $[6,(3.1)]$ and the equivalence of (i) and (iii) in $[6,(2.27)]$ as corollaries to Theorem 1.6.

2. Let $A$ be a Krull domain with quotient field $k$ and let $M$ be a torsion-free $A$-module. Form the symmetric algebra $S_{A}(M)$. For each $p \in \mathcal{P}(A), S(M)_{p}$ $=S_{A_{p}}\left(M_{p}\right)$ is a graded subring of the polynomial ring $S_{k}(M \otimes k)$, since $M_{p}$ is flat over $A_{p}$. Letting $T=\bigcap_{p \in \mathcal{P}(A)} S(M)_{p}$, we find that $T$ is a graded domain with $A$ as its homogeneous component of degree zero. $A \subseteq T$ is thus an inert extension.

Proposition 2.1. In the notation of the above paragraph, if $T$ is either a Krull domain or a Krull $A$-module, then $T_{p}=S(M)_{p}$ is a UFD for each $p \in \mathscr{P}(A)$.

Proof. $S(M)_{p}$ is a UFD if and only if it is a factorial $A_{p}$-module. This holds trivially if $T$ is a Krull $A$-module. If $T$ is a Krull domain, then $S(M)_{p}=T_{p}$ satisfies the ascending chain condition on principal ideals (a.c.c.p.). Since $A_{p}$ is a DVR, $S(M)_{p}$ is flat over $A_{p}$ and this with a.c.c.p. makes $S(M)_{p}$ a factorial $A_{p}$-module [5], [11].

Corollary 2.2. Let $A$ be a Krull domain, $M$ a torsion-free $A$-module, and $T=\bigcap_{p \in \mathcal{P}(A)} S(M)_{p}$. Then $T$ is a Krull domain if and only if it is a Krull $A$ module, in which case $\mathrm{Cl}(T) \cong \mathrm{Cl}(A)$.

Corollary 2.3. Let $A$ be a Krull domain, $M$ a finitely generated torsion-free $A$-module, and $T=\bigcap_{p \in \mathscr{P}(A)} S(M)_{p}$. Then $T$ is a Krull domain and $\mathrm{Cl}(T)$ $\cong \mathrm{Cl}(A)$.

Proof. It suffices to show that $T$ is a Krull $A$-module. Each $S(M)_{p}$ is a polynomial ring over $A_{p}$, hence is factorial over $A_{p}$. We only need to check that the intersection $\cap S(M)_{p}$ of factorial $A_{p}$-modules is locally finite. Now we may choose a finitely generated free module $F$ and an element $a \in A, a \neq 0$, such that $a F \subseteq M \subseteq F$. Then $S(a F) \subseteq T \subseteq S(F)$. S(F) being a free $A$ module is a Krull module. Hence each nonzero element of $T$ is primitive in $S(F)_{p}$ for all but a finite number of $p \in \mathcal{P}(A)$. But $a$ is a unit in $A_{p}$ for all but a finite number of $p \in \mathscr{P}(A)$ and, hence, $T_{p}=S(F)_{p}$ for all but a finite number of $p \in \mathscr{P}(A)$.

We conclude with some examples.

ExAMPLE 2.4. A symmetric algebra $B=S_{A}(M)$ which is a Krull domain such that (PDE) fails for $A \subseteq B$ and $\mathrm{Cl}(A) \cong \mathrm{Cl}(B)$. Let $k$ be a field and $t_{1}, t_{2}$ indeterminates over $k$. Set

$$
A=k\left[t_{1}, t_{2}\right]_{\left(t_{1}, t_{2}\right)} \text { and } B=A[x, y] /\left(t_{1} x+t_{2} y\right)
$$

Then $B$ is the symmetric algebra of the maximal ideal of $A$. The example is due to Samuel [13]; the fact that (PDE) fails and the class groups are not isomorphic was noted by Fossum [7, §10].

EXAmple 2.5. A symmetric algebra $B=S_{A}(M)$ over a Krull domain $A$ such that $B$ is an integral domain, $T=\cap B_{p}$ is a Krull domain, but $B$ is not a Krull 
domain. Let $k$ be a field and $x, y, z$ be indeterminates. Let $A=k[x, y]$ and let $M$ be the ideal of $A$ generated by $x^{2}$ and $y^{2}$. Then $B=S_{A}(M) \cong A\left[x^{2} z, y^{2} z\right]$ [2], [10]. Now $B$ is noetherian, but $x y z \notin B$ although $(x y z)^{2}-\left(x^{2} z\right)\left(y^{2} z\right)=0$. Thus $B$ is not Krull. On the other hand, $T \cong A[z]$ is Krull.

The next example shows that we are not able to weaken the condition in Theorem 1.4 that $B_{p}$ be a UFD for each $p \in \mathscr{P}(A)$.

EXAMPLE 2.6. A graded ring over a DVR which is a Krull domain and a Krull module, but not a UFD. Let $V$ be a DVR, $x$ and $y$ indeterminates assigned degree 1 and $T=V\left[x^{2}, x y, y^{2}\right] . T$ is a Krull $V$-module since it is a free $V$-module. To show that $T$ is a Krull domain note that with $K[u, 1 / u]$ the homogeneous quotient ring of $V[x, y], T=V[x, y] \cap K\left[u^{2}, 1 / u^{2}\right]$, the intersection of two Krull domains. $T$ is not a UFD since $(x y)^{2}=x^{2} y^{2}$.

Acknowledgement. We wish to thank Professor Paul Eakin for many helpful comments and suggestions.

\section{REFERENCES}

1. M. F. Atiyah and I. G. MacDonald, Introduction to commutative algebra, Addison-Wesley, Reading, Mass. 1969. MR 39 \# 4129.

2. J. Barshay, Graded algebras of powers of ideals generated by A-sequences, J. Algebra 25 (1973), 90-99. MR 48 \#11074.

3. P. M. Cohn, Bezout rings and their subrings, Proc. Cambridge Philos. Soc. 64 (1968), 251-264. MR 36 \#117.

4. D. L. Costa, Symmetric algebras and retracts, Dissertation, Univ. of Kansas, 1974.

5. Unique factorization in modules and symmetric algebras, Trans. Amer. Math. Soc. (to appear).

6. P. Eakin and J. Silver, Rings which are almost polynomial rings, Trans. Amer. Math. Soc. 174 (1972), 425-449. MR 46 \#9028.

7. R. M. Fossum, The divisor class group of a Krull domain, Springer-Verlag, New York and Berlin, 1973.

8. R. Gilmer, Multiplicative ideal theory, Dekker, New York, 1972.

9. I. Kaplansky, Commutative rings, Allyn and Bacon, Boston, 1970. MR 40 \#234.

10. A. Micali, P. Salmon and P. Samuel, Intégrité et factorialité des algèbres symmétriques, Proc. Fourth Brazilian Math. Colloq. (1963), Conselho Nacional de Pesquisas, São Paolo, 1965, pp. 61-75. (Portuguese) MR 34 \#7556.

11. A.-M. Nicolas, Modules factoriels, Bull. Sci. Math. (2) 95 (1971), 33-52. MR 44 \# 1653.

12. Extensions factorielles et modules factorable, Bull. Sci. Math. 98 (1974), 117-143.

13. P. Samuel, Anneaux gradués factoriels et modules réflexifs, Bull. Soc. Math. France 92 (1964), 237-249. MR 32 \#160.

14. Lectures on unique factorization domains, Tata Institute Lectures on Math., no. 30, Tata Inst. of Fund. Res., Bombay, 1964. MR 35 \#5428.

Department of Mathematics, University of Virginia, Charlottesville, Virginia 22903

Department of Mathematics, University of Kentucky, Lexington, Kentucky 40506 